\title{
Brain networks for engaging oneself in positive-social emotion regulation
}

\author{
Yury Koush ${ }^{\mathrm{a}, \mathrm{b}, \mathrm{c}, *}$, Swann Pichon ${ }^{\mathrm{d}, \mathrm{e}, \mathrm{f}}$, Simon B. Eickhoffo, h, Dimitri Van De Ville ${ }^{\mathrm{b}, \mathrm{c}}$, Patrik Vuilleumier ${ }^{\mathrm{d}, \mathrm{e}}$, \\ Frank Scharnowski ${ }^{\mathrm{a}, \mathrm{b}}, \mathrm{i}, \mathrm{j}, \mathrm{k}, 1$ \\ ${ }^{a}$ Department of Radiology and Biomedical Imaging, Yale University, 300 Cedar Street, New Haven, CT, 06519, USA \\ ${ }^{\mathrm{b}}$ Institute of Bioengineering, Ecole Polytechnique Fédérale de Lausanne (EPFL), Campus Biotech, Chemin des Mines 9, 1202, Geneva, Switzerland \\ ${ }^{\mathrm{c}}$ Department of Radiology and Medical Informatics, University of Geneva, Geneva, Switzerland \\ ${ }^{\mathrm{d}}$ Geneva Neuroscience Center, Department of Neuroscience, University of Geneva, Case Postale 60, 1211, Geneva, Switzerland \\ ${ }^{\mathrm{e}}$ NCCR Affective Sciences, University of Geneva, Campus Biotech, Chemin des Mines 9, 1202, Geneva, Switzerland \\ ${ }^{\mathrm{f}}$ Faculty of Psychology and Educational Science, University of Geneva, FPSE - 40, Boulevard du Pont-d'Arve, 1211, Geneva, Switzerland \\ ${ }^{g}$ Institute of Neuroscience and Medicine, Brain \& Behaviour (INM-7), Research Center Jülich, 52425, Jülich, Germany \\ ${ }^{\mathrm{h}}$ Institute of Systems Neuroscience, Medical Faculty, Heinrich Heine University Düsseldorf, Düsseldorf, Germany \\ ${ }^{i}$ Department of Psychiatry, Psychotherapy and Psychosomatics, Psychiatric Hospital, University of Zürich, Lenggstrasse 31, 8032, Zürich, Switzerland \\ ${ }^{j}$ Neuroscience Center Zürich, University of Zürich and Swiss Federal Institute of Technology, Winterthurerstr. 190, 8057, Zürich, Switzerland \\ ${ }^{\mathrm{k}}$ Zürich Center for Integrative Human Physiology (ZIHP), University of Zürich, Winterthurerstr. 190, 8057, Zürich, Switzerland \\ ${ }^{1}$ Department of Basic Psychological Research and Research Methods, Faculty of Psychology, University of Vienna, Liebiggasse 5, 1010, Vienna, Austria
}

\section{A R T I CLE IN F O}

Keywords:

Emotion regulation

Positive-social emotions

Self-referential

Connectivity

Meta-analysis

\begin{abstract}
A B S T R A C T
Positive emotions facilitate cognitive performance, and their absence is associated with burdening psychiatric disorders. However, the brain networks regulating positive emotions are not well understood, especially with regard to engaging oneself in positive-social situations. Here we report convergent evidence from a multimodal approach that includes functional magnetic resonance imaging (fMRI) brain activations, meta-analytic functional characterization, Bayesian model-driven analysis of effective brain connectivity, and personality questionnaires to identify the brain networks mediating the cognitive up-regulation of positive-social emotions. Our comprehensive approach revealed that engaging in positive-social emotion regulation with a self-referential first-person perspective is characterized by dynamic interactions between functionally specialized prefrontal cortex (PFC) areas, the temporoparietal junction (TPJ) and the amygdala. Increased top-down connectivity from the superior frontal gyrus (SFG) controls affective valuation in the ventromedial and dorsomedial PFC, self-referential processes in the TPJ, and modulate emotional responses in the amygdala via the ventromedial PFC. Understanding the brain networks engaged in the regulation of positive-social emotions that involve a first-person perspective is important as they are known to constitute an effective strategy in therapeutic settings.
\end{abstract}

\section{Introduction}

Oscar Wilde writes: "I don't want to be at the mercy of my emotions. I want to use them, to enjoy them, and to dominate them." (The Picture of Dorian Gray, 1891). Although these words are overly emphatic, they describe the capacity to maintain, suppress, or change the nature and the intensity of our emotions (Gross, 2002). Emotion regulation allows us to adaptively cope with negative and positive events, and constitutes an important aspect of our personal well-being and social interactions.
Failure to do so is a major risk factor associated with mood and anxiety disorders (Disner et al., 2011; Treadway and Zald, 2011). The psychological and the neural processes underlying emotion regulation have been intensely studied over the past decade, leading to the formulation of brain-based models of emotion regulation (Ochsner and Gross, 2005; Ochsner et al., 2012; Schlosser et al., 2008).

According to these models, successful emotion regulation is strongly dependent on the modulation of stimulus-driven bottom-up responses by cognitive top-down processes (Braunstein et al., 2017; Gross, 2002).

\footnotetext{
* Corresponding author. Department of Radiology and Biomedical Imaging, Yale University, 300 Cedar Street, New Haven, CT, 06519, USA. Email address: yury.koush@yale.edu (Y. Koush)
} 
Specifically, the prefrontal cortex (particularly the dorsomedial prefrontal cortex, dmPFC) is thought to exert influence onto other frontal and limbic regions involved in emotion processing, such as the ventromedial PFC (vmPFC) as well as the amygdala, to which the dmPFC and vmPFC are both densely connected (Banks et al., 2007; Braunstein et al., 2017; Etkin et al., 2011; Kim and Hamann, 2007; Ochsner and Gross, 2005; Ochsner et al., 2012). The vmPFC, in particular, is involved in evaluating and updating the affective value of stimulus representations depending on the context in order to support adequate responses to them (Braunstein et al., 2017; Roy et al., 2012). Although all models of emotion regulation assume the existence of top-down regulation processes between prefrontal regions and the limbic system, most fMRI studies have focused on the down-regulation of negative emotions, and far less have investigated brain connectivity while participants engage themselves in the up-regulation of positive social emotions.

The neural mechanisms of emotion regulation have mainly been investigated using cognitive reappraisal as an explicit cognitive strategy to decrease negative emotions (Braunstein et al., 2017; Ochsner et al., 2012). Reappraisal involves either reinterpreting the meaning of an emotional stimulus, or creating a subjective distance from it (Gross, 2002). Engaging in positive emotions rather than inhibiting or distancing oneself from negative emotions has received far less attention. Interestingly, some of the few existing studies seem to suggest that brain regions involved in the regulation of positive and negative emotions partly overlap (Kim and Hamann, 2007; Ochsner et al., 2012; Vrticka et al., 2012). A better understanding of the mechanisms involved in positive emotion regulation is important because they may not only promote recovery during cognitive therapy of mood disorders (van der Velden et al., 2015), but also facilitate creative thinking, decision-making, problem solving, and social bounds (Carpenter et al., 2013; Nadler et al., 2010). Conversely, their impairment contributes to the severity of mood disorders, particularly anhedonic symptoms associated with depression (Disner et al., 2011; Treadway and Zald, 2011).

Another key element of emotional experiences is that they are often self-referential which is an aspect that determines their intensity irrespective of valence or type of emotion (Scherer, 1982). Adopting a self-relevant egocentric perspective improves emotion regulation success in therapeutic settings, compared to adopting a distant third-person observer position (Holmes et al., 2008). Previous studies have identified the temporoparietal junction (TPJ), dmPFC and precuneus as the core neural system involved in mentalizing (Spreng et al., 2009), mental-state attribution (Teufel et al., 2010), self-referential processing and assessment of the emotional states in social situations (Buckner and Carroll, 2007; Reeck et al., 2016; Zaki and Ochsner, 2012). Impairments of the self-referential neural system is often associated with depression (Lemogne et al., 2009; Wagner et al., 2015), schizophrenia (Harvey et al., 2013) and autism (Murdaugh et al., 2014; Oberman and Ramachandran, 2007; Zaki and Ochsner, 2012) disorders.

Finally, emotions are often tied to social situations as interpersonal interactions constitute an important aspect of humans who are highly social beings (Adolphs, 2003, 2009; Dunbar, 1998). Socially significant stimuli are likely also emotionally relevant, guiding our behavior and communication. Socially relevant stimuli are processed preferentially (e.g., (Hariri et al., 2002)) and recruit brain regions that are inherently linked with emotion processing (e.g., (Reeck et al., 2016; Vrticka et al., 2012)). Frequently, psychiatric conditions are associated with prominent dysfunctional processing of social information and inadequate social behavior (Kennedy and Adolphs, 2012).

The present study sets out to provide new insights into the functions and the dynamics between prefrontal, temporoparietal and limbic regions during the self-referential regulation of positive-social emotions. For this, we applied an emotion regulation paradigm that requires engaging in a positive-social setting with a first-person perspec- tive. Specifically, we contrasted situations where participants passively viewed images depicting positive-social situations, and situations where they engaged themselves in the depicted positive-social situations. To better understand the neural underpinnings of such emotion regulation, we combined complementary brain activation, meta-analytic functional profile and functional as well as effective connectivity analyses. Specifically, using functional magnetic resonance imaging (fMRI), we first identified brain regions involved in the regulation of positive-social emotions and determined their functional profiles using quantitative functional characterization based on meta-analytic procedures (Fox et al., 2005). To systematically investigate the interactions between relevant brain regions, we performed a comprehensive analysis of functional connectivity based on complementary meta-analytic connectivity modeling (MACM, (Fox et al., 2014)) and dynamic causal modeling (DCM, Friston et al., 2003). Furthermore, we used standardized psychometric questionnaires to characterize how specific personality traits and states are linked to the neural dynamics associated with regulating positive emotions. Finally, we integrate the converging results from these complementary methods into a network model that incorporates the function and the dynamic interactions between regions relevant for engaging oneself in positive-social emotion regulation.

\section{Materials and methods}

\subsection{Participants}

Twenty-five healthy human volunteers gave written informed consent to participate in the experiment, which was approved by the local ethics committee. Of the 25 volunteers, two participants had to be excluded due to excessive head movements, leaving twenty-three participants for the analyses (12 male, 11 female, age $27.7 \pm 6.2$ years). All participants were recruited from the local student/research community and had normal or corrected-to-normal vision, as well as no prior history of neurological or psychiatric diseases. Participants were paid 20 CHF/hour for their participation.

\subsection{Experimental design}

The fMRI experiment was set up as a $2 \times 2$ factorial design with factors 'stimuli' and 'task'. The factor 'stimuli' contrasted positive-social scenes (positive images of scenes with people) and neutral scenes of objects (neutral images). The factor 'task' contrasted trials where participants passively viewed the presented images (passive viewing), to trials where they imagined to positively experience the positive-social and the neutral scene from a first-person perspective (self-referential emotion regulation). During the passive viewing condition, participants were asked to focus on the content of the depicted images, and to avoid mind-wandering. During the self-referential emotion regulation condition with positive-social scenes, participants were asked to imagine experiencing the depicted positive-social situation from a first-person perspective, for example, by interacting with the people in an active and pleasant manner. During the self-referential emotion regulation condition with neutral images, participants were asked to imagine themselves using the depicted neutral objects in an active and pleasant manner from a first-person perspective, for example, carrying out the favorite activity with the object. As an objective measure of compliance with these instructions, we asked participants to rate the vividness of their imagery and if they were focus or absent-minded after each fMRI session (on a scale from 1 to 5 ).

To assess the effects of self-referential up-regulation of positive-social emotions on the brain and to identify personality traits that might predict changes in brain connectivity related to emotion regulation, participants were asked before and after the fMRI experiment to complete the Emotion Regulation Questionnaire (ERQ, (Gross and John, 
2003)), the Positive And Negative Affect Schedule (PANAS-P, (Watson et al., 1988)) and the Beck Depression Inventory (BDI, (Beck et al., 1961)). These questionnaires measure habitual use of cognitive reappraisal and expressive suppression as emotion regulation strategies (ERQ), general affective state (PANAS-P), and severity of depression symptoms (BDI), respectively.

We also asked participants to rate valence and arousal of 60 positive and 60 neutral images (i.e. 30 images before and 30 images after the experiment) that were similar to the ones used during the experiment (i.e., their IAPS valence and arousal values did not differ from those of the experiment, two-tailed paired t-tests, p-values $>.20$ ) using the standard self-assessment manikin ratings (SAM) on the IAPS 9-point rating scale (Lang et al., 1993).

During the fMRI experiment, participants performed two runs of 11.3 min each. Each of the two runs consisted of 2 blocks, with a block being either the passive viewing or the self-referential emotion regulation condition. Before a block started, either the instruction 'view' or 'regulate' was shown to the participant for $4 \mathrm{~s}$. The order of passive viewing and emotion regulation blocks was randomized across participants. Each block consisted of seven $24 \mathrm{~s}$ periods during which positive images were presented, and seven $24 \mathrm{~s}$ period during which neutral images were presented. The order of positive and neutral periods was randomized across participants, and the images were presented at a rate of 4 images per period (6s display duration). These presentation times were chosen to facilitate imagery during the emotion regulation runs (Ochsner et al., 2009; Vrticka et al., 2012).

\subsection{MRI data acquisition}

The experiment was performed on a 3T MRI scanner (Trio Tim, Siemens Medical Solutions, Erlangen, Germany) at the Brain and Behavior Laboratory (University of Geneva). At the beginning of the scanning session we acquired for each participants a T1-weighted structural image (32 channel receive head coil, 3D MPRAGE, voxel size $=1 \mathrm{~mm}^{3}$ isotropic, flip angle $\alpha=9^{\circ}$, TR $=1900 \mathrm{~ms}$, TI $=900 \mathrm{~ms}$, TE $=2.27 \mathrm{~ms}$ ), and a double-echo gradient-echo field maps (TE1 $=5.19 \mathrm{~ms}$, $\mathrm{TE} 2=7.65 \mathrm{~ms}$, voxel size $=3 \times 3 \times 2.2 \mathrm{~mm}^{3}$, TR $=456 \mathrm{~ms}$, flip angle $=60^{\circ}$, bw $=260 \mathrm{~Hz} /$ pixel). Functional images were acquired with a single-shot gradient-echo T2*-weighted EPI sequence with 345 repetitions (TR $=2050 \mathrm{~ms}, 32$ slices volume, matrix size $=120 \times 120$, voxel size $=2 \times 2 \times 2 \mathrm{~mm}^{3}$, slice-gap distance factor $=25 \%$, flip angle $=75^{\circ}, \mathrm{bw}=1.57 \mathrm{k} \mathrm{H} \mathrm{z} /$ pixel, $\mathrm{TE}=35 \mathrm{~ms}$, GRAPPA, iPAT $=3$ ). The field of $v$ iew (FOV) is illustrated in Fig. 1 . The positive phase-encoding polarity and the slice tilt of $\sim-15^{\circ}$ in combination with relatively high spatial resolution at $3 \mathrm{~T}$ was chosen to ensure optimal sensitivity and precise segregation of frontal and limbic brain areas (Weiskopf et al., 2006, 2007). The GRAPPA acceleration factor was chosen to achieve a compromise between the SNR of the 32 channel coil at 3T (Triantafyllou et al., 2011) and a reasonable temporal resolution required for DCM estimations (Stephan et al., 2010). Heart rate and respiration were continuously monitored throughout the experiment with a modular data acquisition system (MP150, $1 \mathrm{kHz}$ sampling rate, BIOPAC Systems Inc). Heart rate was measured using a pulse oximetry sensor, and respiration was measured using an elastic belt around the participant's chest. Visual stimuli and instructions were displayed using a rectangular projection screen at the rear of the scanner bore with a mirror positioned within the head-coil. All participants were instructed to breathe steadily and to remain as still as possible. To account for the sensitivity of especially the functional connectivity analysis to head motion artifacts, we excluded two participants that exceeded an established threshold of 25 head displacements (Van Dijk et al., 2012). The number of head movements was counted as the number of relative displacements $>.1 \mathrm{~mm}$, with displacements being estimated as the root-mean-square of the volumetric translation parameters combined (Van Dijk et al., 2012).

\subsection{Stimuli}

We used as stimuli two sets of photographs (172 photographs each) from the International Affective Picture Set (IAPS, (Lang et al., 1993)), the Nencki Affective Picture System (NAPS, (Marchewka et al., 2013)) and the Geneva Affective Picture Database (GAPED, (Dan-Glauser and Scherer, 2011)). Images in the first set depicted positive-social situations (positive images of scenes with people; mean normative valence $6.97 \pm 0.68$, mean normative arousal $4.97 \pm 0.82$ ). Images in the second set depicted non-social neutral scenes and objects (mean norma-

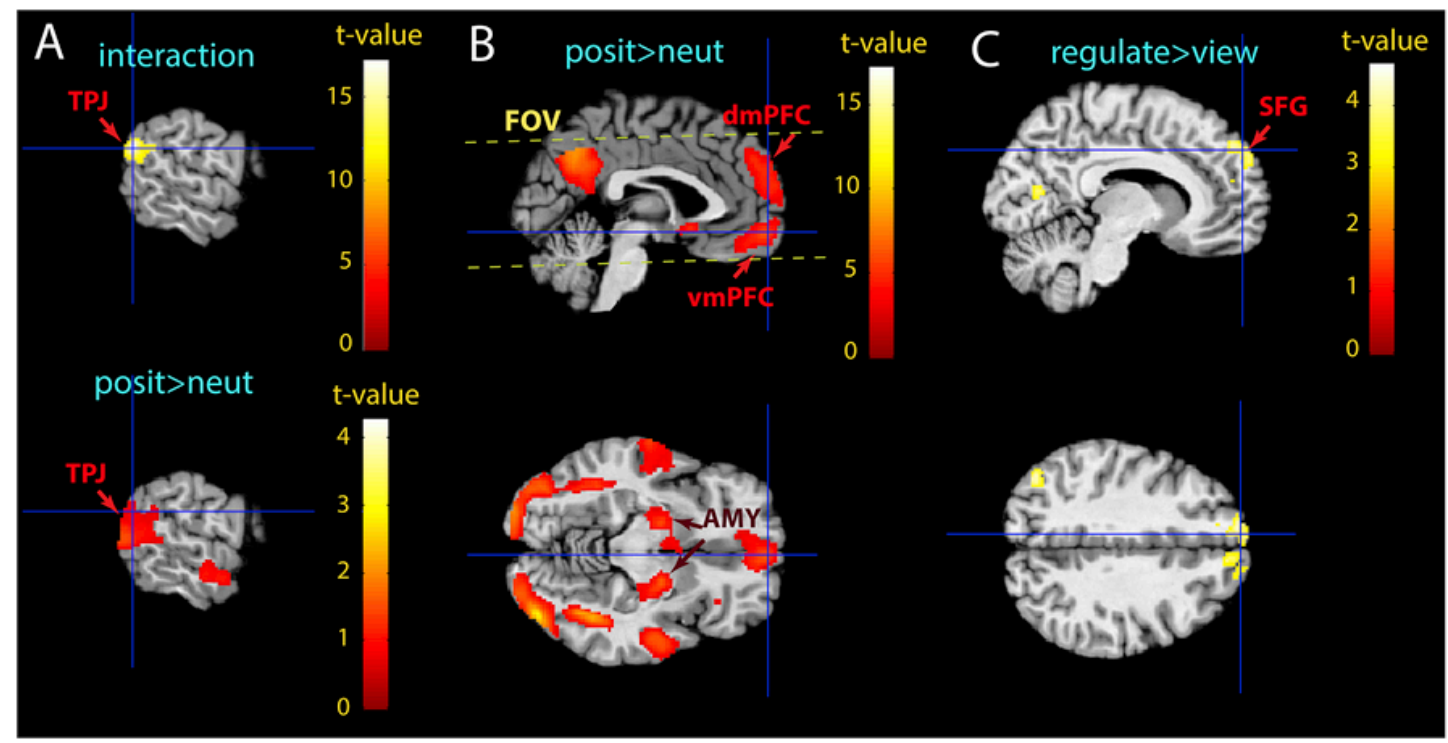

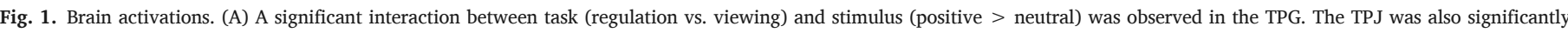

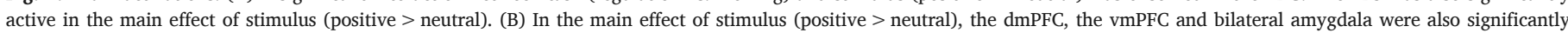

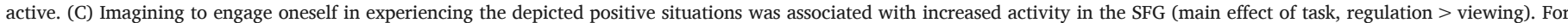

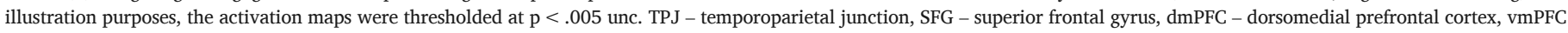
- ventromedial prefrontal cortex, AMY - amygdala, FOV - field of view. 
tive valence $5.21 \pm 0.60$, mean normative arousal $3.52 \pm 1.05$ ). From each set, 60 images were used for the pre- and post-scanning behavioral ratings (positive images: mean normative valence $7.06 \pm 0.52$, mean normative arousal $5.02 \pm 0.95$ ). The remaining 112 images from the first set (together with the 112 images from the second set) were used for the fMRI scans (positive images: mean normative valence $6.92 \pm 0.74$, mean normative arousal $4.94 \pm 0.75$ ). There were no valence and arousal differences between images used for the fMRI scans and images used for the behavioral ratings, nor between images used for the pre-compared to the post-scanning behavioral ratings (all p-values $>.20$ ). The order of image presentation for the fMRI scans and for the behavioral ratings was pseudo-randomized for each participant, and each image was shown only once to a participant. This ensured a uniform distribution of stimuli valence and arousal in the picture subsets and balanced potential color, intensity and scenery differences between pictures from the different databases. The images were presented centrally and had a diameter of $\sim 12^{\circ}$ visual angle. Positive images were framed in green, and neutral images were framed in white.

\subsection{Data analysis}

The fMRI data analysis was performed using SPM12 (Wellcome Trust Center for Neuroimaging, Queen Square, London, UK). The first 5 EPI volumes were discarded to account for T1 saturation effects. The remaining images were spatially realigned to the mean scan of each session, coregistered to the standard MNI structural template using DARTEL (Ashburner, 2007), corrected for geometric distortions (Jenkinson, 2003), and smoothed with an isotropic Gaussian kernel with moderate $6 \mathrm{~mm}$ full-width-at-half-maximum (FWHM). For the subject-level analysis, we specified general linear models (GLM) with 4 regressors for the four conditions (i.e. viewing-neutral, viewing-positive, regulation-neutral, regulation-positive), and 6 covariates derived from head movement parameters to capture residual motion artifacts. The regressors were modeled as boxcar functions convolved with the canonical hemodynamic response function (HRF) in SPM12.

For the whole-brain group level analysis, we performed a factorial 2-way ANOVA with fixed factor 'condition' (viewing-neutral, viewing-positive, regulation-neutral, regulation-positive) and random factor 'subject'. As covariates, we included the participants' age and gender. Statistical maps were corrected for multiple comparisons using whole-brain family-wise error correction (FWE, $\mathrm{p}<.05$ ). Small volume correction (SVC) at the peak-level (FWE, $\mathrm{p}<.05$ ) was applied to the temporoparietal junction (TPJ, interaction contrast map, $\mathrm{p}<.005$ unc.) and superior frontal gyrus (SFG, regulation $>$ viewing contrast map, $\mathrm{p}<.005$ unc.) using a sphere of $10 \mathrm{~mm}$ radius centered on independent coordinates defined with Neurosynth database (MNI coordinates for association test, entry 'self-referential' for TPJ, [-50, -56, 30], and entry 'emotion regulation' for SFG, [-12 58 38]).

\subsection{ROI definitions}

All ROIs except the amygdala were defined as spheres with a $10 \mathrm{~mm}$ radius around the corresponding cluster center of gravity. The centers of gravity were defined for the clusters of the thresholded activation maps ( $<.005$ unc.) as implemented in the Anatomy toolbox (Eickhoff et al., 2005). The ROIs did not overlap. The dmPFC, vmPFC and SFG centers of gravity were defined in the positive $>$ neutral images contrast for the $\operatorname{vmPFC}([1,52,-13])$ and the dmPFC $([2,57$, 24]) clusters, and in the regulation $>$ viewing contrast for the SFG (left: [- $\left.\begin{array}{lll}2 & 52 & 35\end{array}\right]$, right: $\left[\begin{array}{lll}10 & 52 & 38\end{array}\right]$ ) clusters. To localize the bilateral TPJ, we masked the positive $>$ neutral contrast with the regulation $>$ viewing contrast (whole-brain FWE correction, $\mathrm{p}<.05$ ), and defined left $[-45,-68,26]$ and right $[50,-57,28]$ centers of gravity for the identified TPJ clusters using the Anatomy toolbox. The bilateral amygdala ROIs were defined anatomically based on the Talairach Daemon atlas (Lancaster et al., 2000) because it is a very small region that is vulnerable to dropout artifacts and for which a spherical ROI would have likely included non-amygdala voxels in close proximity.

For each ROI, we first extracted the first principal component of the individual local multivariate time-series using singular value decomposition to account for potential functional heterogeneity (Friston et al., 2006; Stephan et al., 2010). The time-series were then corrected for signal drift and head motion artifacts using a GLM model with regressors for conditions, head movement covariates, and linear drift. In addition, the high frequency noise and spikes were removed using a modified Kalman filter (Koush et al., 2012).

\subsection{Analyses of psychometric questionnaires and ratings}

We calculated two-tailed Spearman rho correlations between the participant's average questionnaires and behavioral ratings (i.e. the average of the scores/ratings from before and after the fMRI experiment was calculated) with their differences of the beta values between the self-referential emotion regulation and the passive viewing conditions of each ROI. This correlation was calculated separately for each ROI. The statistical significance was thresholded at $\mathrm{p}<.05$ and corrected for multiple comparisons using false-discovery-rate (FDR). The correlations were z-scored for illustration purposes.

\subsection{Meta-analytic quantitative functional characterization and connectivity analysis}

We characterized the functions of activated voxels in our ROIs using the BrainMap database (Fox et al., 2005), which contains the results of a large number of neuroimaging experiments as coordinates of the maxima of the statistical maps and associated meta-data employing taxonomic categories (cognition, emotion, perception, action, interoception), the experimental paradigm classes, and their related subcategories (Fox et al., 2005). Two types of inference can be drawn on the func-tional characterization test, i.e., forward and reverse inference. Forward inference describes how likely a region is activated given a particular psychological category, reverse inference describes how likely a partic-ular psychological category is given activation in that region. We first defined seed regions representing our ROIs as activation clusters identi-fied by the group-level analysis. We then used forward inference to determine the probability of observ-ing activity in these seed regions given a specific psychological category or paradigm class (Bzdok et al., 2013; Yarkoni et al., 2011). Note that BrainMap uses the general categories if no specific sub-category can be assigned.

To characterize the co-activation patterns between our ROIs, we performed a meta-analytic connectivity analysis (MACM), which computes the brain-wide co-activation pattern for each seed cluster (Fox et al., 2014). For this analysis, ROI seed regions were entered into a meta-analytic connectivity modeling analysis, which computes the brain-wide co-activation pattern for each seed cluster (Fox et al., 2014). Then, for each ROI, activation likelihood estimation (ALE) meta-analyses were performed on the sets of coordinates identified as co-activated with the respective ROI (Laird et al., 2005). The resultant ALE maps were thresholded at $\mathrm{p}<.05$ and corrected for multiple comparisons using FWE.

\subsection{Effective connectivity analysis}

DCM is a model-based effective connectivity analysis which provides a Bayesian framework to model functional brain networks as a 
set of differential equations that describe not only the architecture of the network (i.e., the ROIs and their connections), but also the dynamic interactions within the network due to external inputs (e.g., the presentation of positive images) and due to contextual modulations (e.g., engaging actively in the depicted positive situations). Using Bayesian model selection (BMS) family-level inference, DCM allows for quantitatively comparing which model architecture explains the observed data best (Penny et al., 2010). DCM also allows for computing a posterior probability of the model parameters and their mean values while taking into account the evidence and complexity of every to-be-averaged model using Bayesian model averaging (BMA), and can thus shed light on the connectivity changes within a network during specific task conditions. Positive parameter values indicate that increasing activity in a region results in increasing rate of change in the connected region, whereas negative parameters indicate that increasing activity in a region results in decreasing rate of change in the connected region. In order to generalize the results of these analysis to the population, we used a fixed effect (FFX) approach across performed experimental runs and a random effect (RFX) approach to account for inter-subject variability. Based on the results from the previous analyses, we included 5 ROIs in our DCM analysis: the bilateral SFG, the vmPFC, the dmPFC, the bilateral TPJ, and the bilateral amygdala.

Due to the large number of possible models within each family, we had to limit the model space by assuming that each connection is bilateral, and that external and modulatory inputs affect both hemispheres in the same way (e.g. the external inputs into the left and right amygdala are identical) (Fig. S3). Also, the models without external stimuli were excluded. Because the TPJ is the only region that showed a significant task $\times$ stimulus interaction, we were particularly interested in the connectivity between the TPJ and the other regions involved in either the task or stimulus processing. Therefore, we varied the models in how the TPJ was connected to these other regions. The first and second model families contained all models with the TPJ connected to the other 4 ROIs, and the amygdala connected to the dmPFC or vmPFC (496 models each). The third to eighth model families contained all models with the TPJ connected to any of the other 3 ROIs, and the amygdala connected either to the dmPFC or vmPFC (248 models each). The ninth to fourteenth model families contained all models with the TPJ connected to any of the other 2 ROIs, and the amygdala connected to either the dmPFC or vmPFC (124 models each). Model families fifteen to twenty-eight were partitioned like model families one to fourteen, except that there was no connection between the bilateral amygdala and TPJ (model families fifteen and sixteen: 248 models each; model families seventeen to twenty-two: 124 models each; model families twenty-three to twenty-eight: 62 models each). The twenty-ninth and thirtieth model families contained all models with the TPJ connected only to the amygdala ( 62 models each). The entire set of models thus comprised 4'960 model alternatives per participant (23 participants), run (2 runs), and condition (2 conditions), resulting in a total of 456'320 DCM estimations which were computed on the Castor and Baobab High Performance Computing (HPC) clusters of the EPFL and the University of Geneva.

Next, we estimated all possible model alternatives and then applied BMS family level inference procedures to investigate which general model structure underlay the passive viewing and the emotion regulation conditions when being presented with positive social scenes (Penny et al., 2010). We partitioned the model space in subsets of thirty model families that differed in the connectivity pattern between our 5 ROIs (Fig. S3). The model families with the highest family exceedance probabilities (i.e., the winning models) for the passive viewing and the emotion regulation conditions were identified using family-level BMS, and their parameters were estimated using BMA. We used DCM as implemented in SPM 12.

\section{Results}

\subsection{Brain activations associated with self-referential positive emotion regulation}

We found a significant interaction (Table 1 , task $\times$ stimuli) in the left TPJ, which was mainly driven by a greater activation difference between the positive and neutral pictures in the self-referential regulation as compared to the passive viewing condition (Fig. 1, Table 1). The main effect of stimulus (positive $>$ neutral images) was associated with significant activations in the bilateral TPJ (Fig. 1A), bilateral amygdala, dmPFC and vmPFC, but also the primary visual cortex (PVC) and lateral occipital cortex (near V5/MT), the lateral fusiform gyrus (around the fusiform face area (FFA)), the precuneus, the superior temporal sulcus (STS), and the inferior frontal gyrus (IFG) (Fig. 1B). The amygdala showed no evidence of functional lateralization (i.e., comparing individual contrast images with their flipped counterparts did not reveal a significant difference). The main effect of task (regulation $>$ viewing) revealed significant activations in the SFG, which did not overlap with dmPFC (Fig. 1C)

The observed brain activations were not related to cardio-respiratory artifacts, i.e. heart rate and respiration showed no difference be-

Table 1

Brain areas related to stimulus content and emotion regulation.

\begin{tabular}{|c|c|c|c|c|c|c|c|}
\hline \multirow[t]{2}{*}{ Contrast } & \multicolumn{2}{|c|}{ Anatomical cluster } & \multicolumn{3}{|c|}{ Main peak MNI coordinates } & \multirow[t]{2}{*}{ T-value } & \multirow[t]{2}{*}{$\mathrm{p}$-value } \\
\hline & & & $x$ & $y$ & $z$ & & \\
\hline Interaction task $\times$ stimuli & TPJ & $\mathrm{L}$ & -58 & -56 & 26 & 4.00 & $.014+$ \\
\hline regulation $>$ viewing & SFG & $\mathrm{L}$ & -14 & 56 & 36 & 3.91 & $.018+$ \\
\hline \multirow[t]{11}{*}{ positive $>$ neutral } & TPJ & $\mathrm{L} / \mathrm{R}$ & $-42 / 44$ & $-54 /-56$ & $24 / 18$ & $3.76 / 10.28$ & $.025+/<.001$ \\
\hline & $\mathrm{dmPFC}$ & $\mathrm{R}$ & 6 & 50 & 34 & 5.48 & .025 \\
\hline & vmPFC & $\mathrm{R}$ & 4 & 58 & -12 & 6.29 & .002 \\
\hline & AMY & $\mathrm{L} / \mathrm{R}$ & $-18 / 18$ & $-8 /-6$ & $-14 /-14$ & $6.28 / 6.94$ & $.002 /<.001$ \\
\hline & V5/MT & $\mathrm{L} / \mathrm{R}$ & $-48 / 48$ & $-72 /-74$ & $12 / 4$ & $10.93 / 16.96$ & $<.001 /<.001$ \\
\hline & PVC & $\mathrm{L} / \mathrm{R}$ & $-22 / 28$ & $-102 /-96$ & $-2 /-6$ & $9.37 / 10.11$ & $<.001 /<.001$ \\
\hline & FFA & $\mathrm{L} / \mathrm{R}$ & $-42 / 42$ & $-52 /-52$ & $-20 /-18$ & $9.52 / 13.43$ & $<.001 /<.001$ \\
\hline & Precuneus & $\mathrm{R}$ & 2 & -58 & 40 & 8.42 & $<.001$ \\
\hline & STS & $\mathrm{L} / \mathrm{R}$ & $-68 / 54$ & $-12 /-10$ & $-12 /-12$ & $5.64 / 6.44$ & $.016 / .001$ \\
\hline & sgACC & $\mathrm{R}$ & 2 & 12 & -8 & 4.95 & $.008^{*}$ \\
\hline & IFG & $\mathrm{R}$ & 36 & 16 & 26 & 5.33 & .041 \\
\hline
\end{tabular}

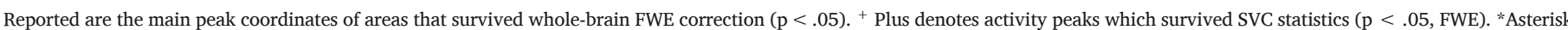

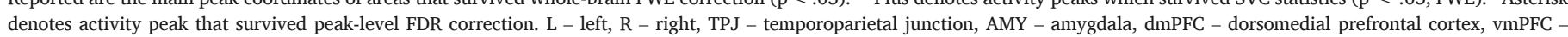

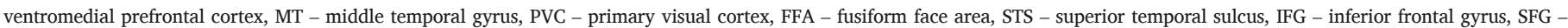
superior frontal gyrus. 
tween the experimental conditions (one-way ANOVA, ps > .05; heart rate: positive scenes regulation $64.92 \pm 3.61 \mathrm{bpm}$ and viewing $63.32 \pm 3.23 \mathrm{bpm}$, neutral scenes regulation $64.87 \pm 3.50 \mathrm{bpm}$ and viewing $63.62 \pm 3.27 \mathrm{bpm}$; respiratory effort: positive scenes regulation $31.92 \pm 4.26$ a.u. and viewing $31.08 \pm 4.34$ a.u., neutral scenes regulation $31.94 \pm 4.28$ a.u. and viewing $31.26 \pm 4.32$ a.u.). Also, the results of the whole-brain group level analysis were robust without including age and gender as covariates: TPJ (interaction, peak $\mathrm{t}=4.35, \mathrm{p}=.008$, SVC-corrected, coordinates $[-60,-58,24]$ ), SFG (regulation $>$ viewing contrast, peak $\mathrm{t}=3.81, \mathrm{p}=.037$, SVC-corrected, coordinates [-12, 54, 36]), dmPFC (positive $>$ neutral contrast, whole-brain FWE-corrected, $\mathrm{t}=3.81, \mathrm{p}=.050$, coordinates $[6,50,34]$ ), vmPFC (positive $>$ neutral contrast, whole-brain FWE-corrected, $\mathrm{t}=5.97, \mathrm{p}=.005$, coordinates $[4$, $58,-12]$ ), AMY(R) (positive > neutral contrast, whole-brain FWE-corrected, $\mathrm{t}=6.93, \mathrm{p}<.001$, coordinates $[18,-6,-14]$ ), AMY(L) (positive $>$ neutral contrast, whole-brain FWE-corrected, $\mathrm{t}=6.21, \mathrm{p}=.002$, coordinates $[-18,-8,-14])$.

\subsection{Behavioral effects of self-referential positive emotion regulation}

All participants strictly adhered to the experimental protocol and reported having engaged in the emotion regulation and passive viewing conditions (mean ability to 'focus': $4.4 \pm 0.8$, mean vividness of imagery: $3.7 \pm 1.1$ ). This engagement led to behaviorally measurable changes. Comparing pre- and post- experiment scores, we found a significant reduction in depression scores (BDI) (before: $4.4 \pm 1.2$; after: $3.4 \pm 1.1 ; \mathrm{t}=3.4, \mathrm{p}<.01$ ), a significant increase in reappraisal scores (ERQ-R) (before: $28.7 \pm 1.2$; after: $30.7 \pm 1.3$; $\mathrm{t}=3.4, \mathrm{p}=.04$ ), and a significant decrease in negative mood (PANAS-N) (before: $26.5 \pm 2.6$; after: $23.0 \pm 2.6 ; \mathrm{t}=3.7, \mathrm{p}<.01$ ) (Fig. $2 \mathrm{~A}$; all one-tailed paired t-tests, FWE-corrected). In contrast, thought suppression scores (ERQ-S) (before: $14.1 \pm 1.0$; after: $14.1 \pm 1.4$ ), positive mood scores ((PANAS-P) before: $60.5 \pm 2.7$; after: $60.4 \pm 3.0$ ), and valence and arousal ratings of emotional stimuli did not show significant differences after compared to before the experiment (Fig. 2A and B; one-tailed paired t-tests, p-values $>.05)$. We also found that higher thought suppression scores (ERQ-S) correlated negatively with activity level increases between emotion regulation and passive viewing in the dmPFC (Fig. 2C; rho $=-0.50$, adjusted $\mathrm{p}=.04$, FDR correction, $\mathrm{q}<0.05$ ) and in the vmPFC (Fig. 2C, rho $=-0.51$, adjusted $\mathrm{p}=.04$, FDR correction, $\mathrm{q}<0.05)$.

\subsection{Meta-analytic quantitative functional characterization}

The final step in characterizing the ROIs in our study was to identify the psychological processes that these areas are associated with us- ing meta-analytic procedures and the BrainMap database. The activation clusters in the bilateral amygdala were predominantly associated with the categories 'Emotion' and 'Perception' (Fig. S1). For the SFG and vmPFC, the dominant category was 'Emotion', followed by 'Cognition'. For the dmPFC and TPJ, the dominant category was 'Cognition', followed by 'Emotion'. For the paradigm classes, the activation clusters in the bilateral amygdala were predominantly associated with 'Olfactory Monitor/Discrimination', 'Classical Conditioning' and 'Affective Pictures' paradigms. The vmPFC was predominantly associated with 'Competition/Cooperation', 'Reward', and 'Face Monitor/Discrimination' paradigms. In contrast, both the dmPFC and the SFG were predominantly associated with 'Self-Reflection', 'Theory of Mind', 'Episodic Recall' and 'Emotion Induction' paradigms. The TPJ was predominantly associated with 'Theory of Mind' paradigms.

\subsection{Connectivity underlying self-referential positive emotion regulation}

Now that we have characterized the ROIs that are associated with engaging oneself in positive emotion regulation, we turn towards the key question about how these ROIs interact. To define an initial network model of functional connectivity for our ROIs, we used MACM. We found a strong overlap of the co-activation maps between seed SFG, dmPFC, vmPFC and TPJ; between seed dmPFC, amygdala and vmPFC; and between seed vmPFC and amygdala indicating that they are functionally highly inter-connected (Fig. S2).

The next critical objective of our study was to formally assess the effective network dynamics between the highly inter-connected ROIs during positive-social emotion regulation using DCM. Given the activity patterns (Fig. 1), the functional characteristics (Fig. S1), and the co-activation maps (Fig. S2), this DCM analysis focused specifically on the interactions between the TPJ, PFC, and amygdala, as well as on the functional subdivisions of the PFC areas. We began by investigating which network structure best explains our data during the passive viewing and the emotion regulation conditions. During passive viewing, the highest model exceedance probability was observed for the model family with direct connections between the TPJ and the other four ROIs, and between the amygdala and the $\mathrm{dmPFC}$, together with highly interconnected prefrontal ROIs ( $\mathrm{Pe}=.29$, Figs. $3 \mathrm{~A}$ and $\mathrm{S} 3$, model family 1$)$. In contrast, during emotion regulation, the dominant model family showed that now the TPJ was not connected with the dmPFC, and the amygdala was connected to the vmPFC ( $\mathrm{Pe}=.44$, Figs. $3 \mathrm{~A}$ and $\mathrm{S} 3$, model family 7). Note that because in our experimental design, the image presentation and the task (i.e., passive viewing or emotion regulation) are inherently linked to one another, the external input can reflect either the image presentation, the task, or both.
A

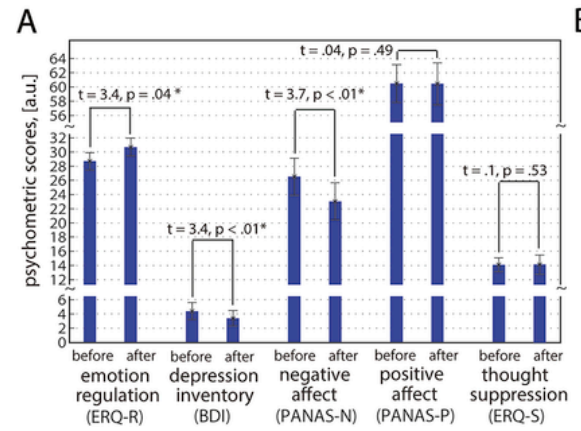

B

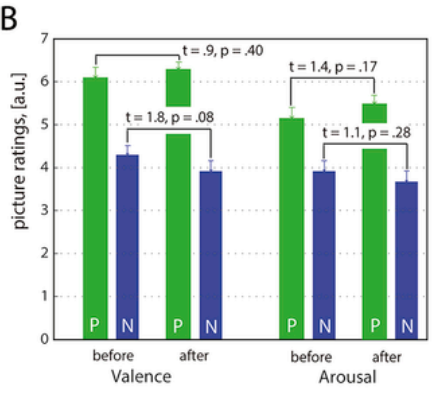

C

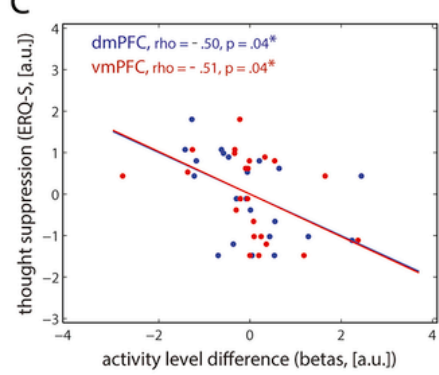

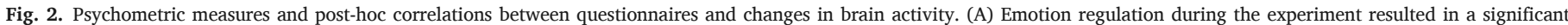

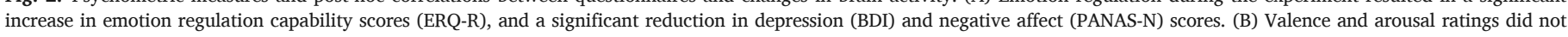

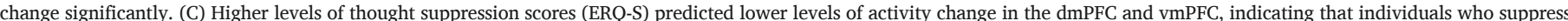

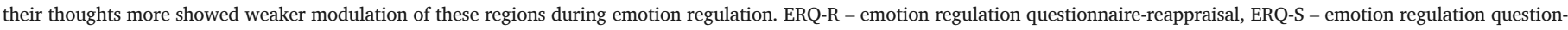

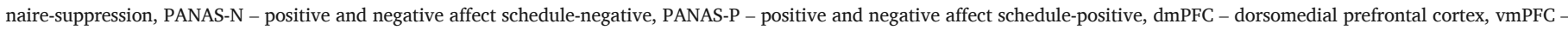
ventromedial prefrontal cortex. * survived FDR correction for multiple comparisons $(\mathrm{q}<.05)$. 
Finally, we compared different parameters of the models from the winning model subfamilies using Bayesian model averaging (BMA). During passive viewing, the TPJ, vmPFC and amygdala responded positively to the external input, while the SFG responded negatively (Fig. 3B). Overall, the ROIs are positively connected, except for the negative connections from the amygdala to the TPJ. During emotion regulation, the vmPFC, dmPFC, SFG and TPJ responded positively to the external input (Fig. 3C). The vmPFC and especially the SFG showed both positive connection strengths onto the TPJ and dmPFC. The vmPFC showed positive connection strengths to the bilateral amygdala. Furthermore, during engaging oneself in positive emotion regulation, the dmPFC decreased the responsiveness of the $\mathrm{VmPFC}$ as indicated by a negative connection strength. Taken together, these findings reveal distinct modes of interactions between prefrontal, temporoparietal and limbic regions during self-engaged positive emotion regulation.

\section{Discussion}

Previous studies of emotion regulation have focused predominantly on reducing negative emotions through the use of cognitive strategies like reappraisal and detachment (Ochsner et al., 2012). The regulation of emotions by positive appraisal and explicitly engaging oneself in positive-social emotions has received far less attention, despite the fact that positive emotions facilitate cognitive performance, creativity, and social bounds (Carpenter et al., 2013; Nadler et al., 2010). Positive emotions are also a key component of healthy mood regulation, and they are distinctly impaired in clinical disorders such as depression and anxiety (Carpenter et al., 2013; Disner et al., 2011; Nadler et al., 2010; Treadway and Zald, 2011). Understanding the mechanism and impact of first-person projections for positive-social emotion regulation are important as they are known to constitute an effective strategy in therapeutic settings (Buckner and Carroll, 2007; Holmes et al., 2008). Here, we combined neuroimaging measures with quantitative functional characterization of the activated areas, meta-analytic and model-driven analyses of brain connectivity to shed light on the dynamics of brain networks mediating the engagement of oneself in the cognitive regulation of positive-social emotions. We also related these processes to individual difference in affective experience and thought control abilities.

Our results revealed that self-referential positive-social emotion regulation recruited a distributed network of prefrontal, temporoparietal, and limbic brain areas. Remarkably, this network was identified through converging evidence from two complementary approaches to studying brain connectivity, namely, meta-analytic functional connectivity analysis using MACM (Fig. S2) and specific model-based effective connectivity analysis using DCM (Figs. 3 and 4). While the regions that comprise this network have previously been shown to be involved in related emotion regulation paradigms (Braunstein et al., 2017; Reeck et al., 2016), we additionally provide a quantitative functional characterization of these regions (Fig. S1) and information about how they effectively interact in a network (Figs. 3 and 4). Integrating these complementary results, we found that during engaging oneself in positive-social emotions, cognitive control processes originate primarily in the

A

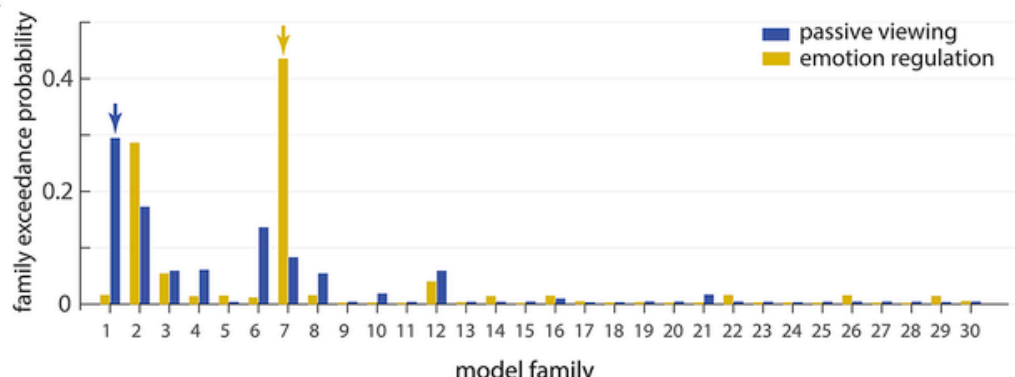

B

winning model family 1 for passive viewing

\section{c}
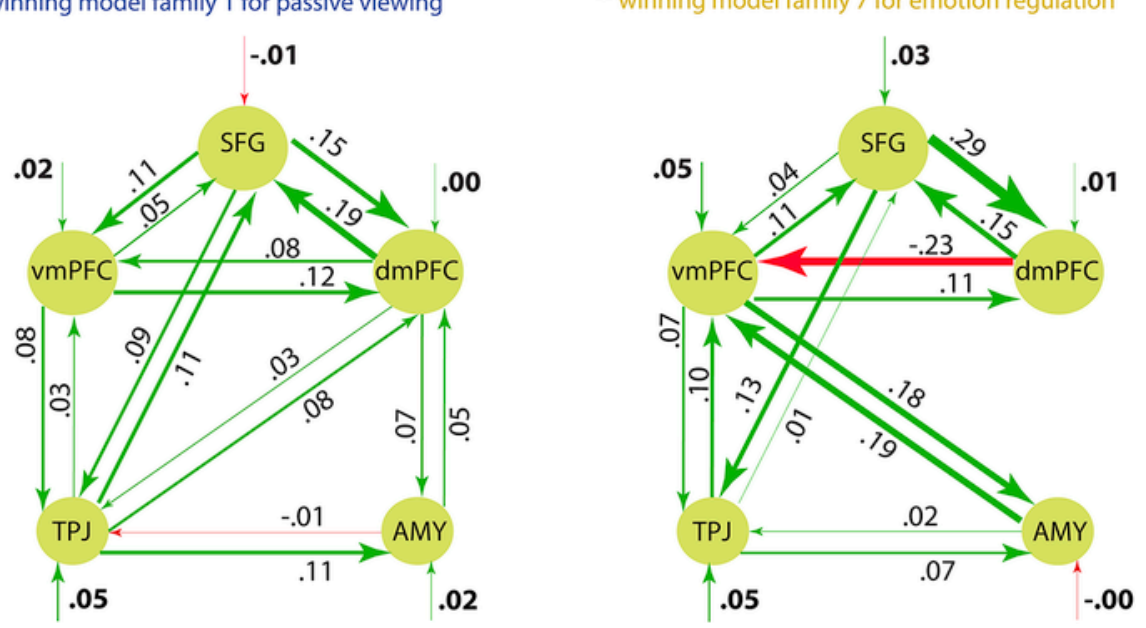

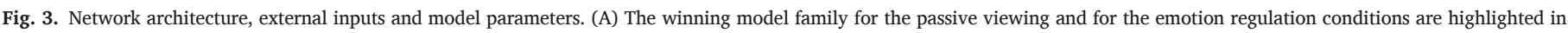

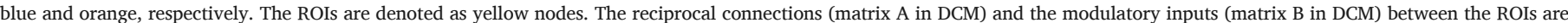

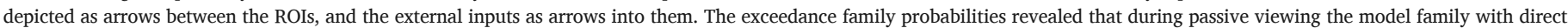

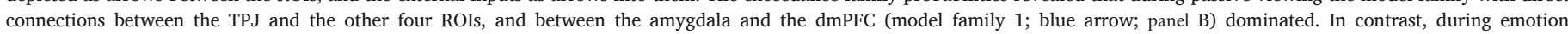

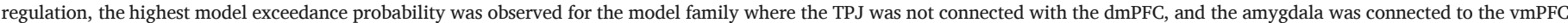

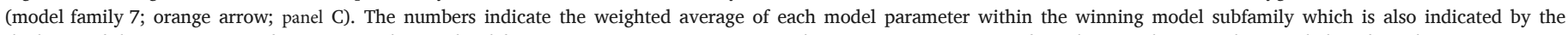

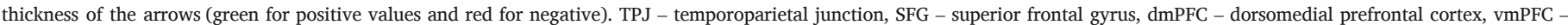
ventromedial prefrontal cortex, AMY - amygdala. (For interpretation of the references to color in this figure legend, the reader is referred to the Web version of this article.) 


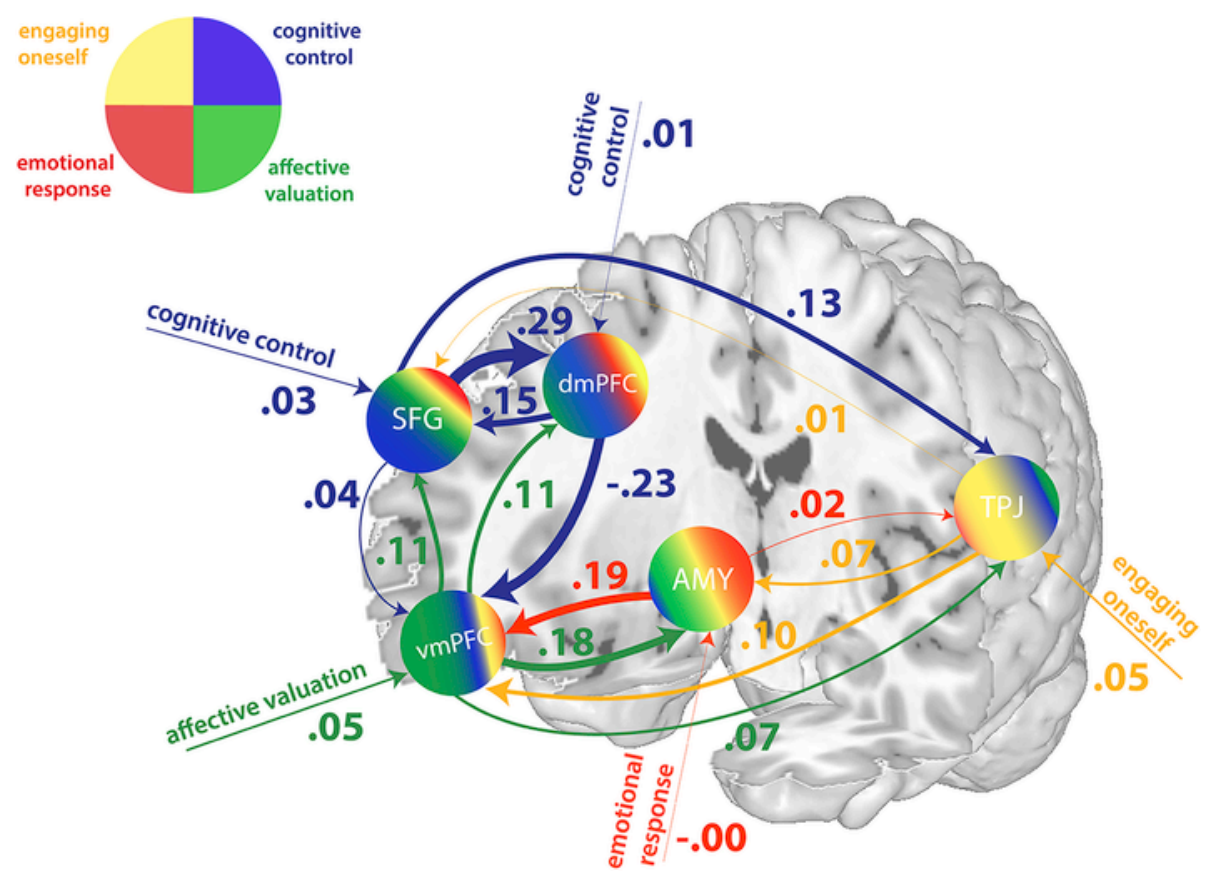

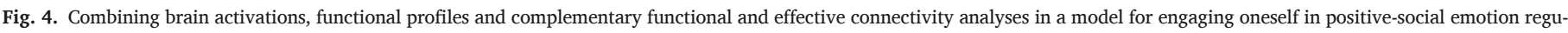

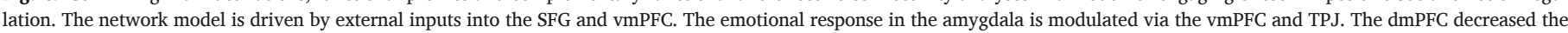

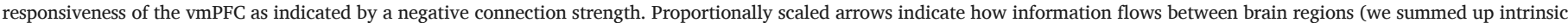

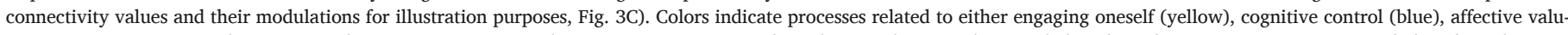

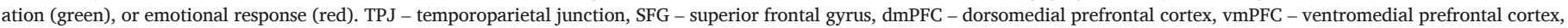
AMY -amygdala. (For interpretation of the references to color in this figure legend, the reader is referred to the Web version of this article.)

SFG (Fig. 4). The SFG then influences the TPJ which is associated with engaging oneself, the dmPFC which mediates cognitive control over the emotional response, and the vmPFC which serves mainly the affective valuation. The affective valuation of the vmPFC is also strongly modulated by the dmPFC, and by inputs from the amygdala as well as the TPJ, which are related to the emotional response and engaging oneself, respectively.

We found significant activation of the left TPJ in the interaction stimuli $\times$ task, which was driven by the fact that the TPJ responded particularly strongly to engaging in positive-social scenes. The TPJ has been shown to be associated with self-referential perspective taking (Saxe and Kanwisher, 2003), assessment of the emotional states in social situations (Buckner and Carroll, 2007; Reeck et al., 2016; Zaki and Ochsner, 2012), bodily self-location and self-consciousness (Ionta et al., 2014). These descriptions match the meta-analytic functional profile of the TPJ activations in our study, which were predominantly 'Cognition' and 'Theory of Mind' (Fig. S1). Also, the lateralization of our findings is echoed by previous findings. For example, the left TPJ is more strongly connected to the executive control network and in particular the dmPFC (Kucyi et al., 2012), and activity especially in the left TPJ is associated with reasoning about the beliefs of others (Samson et al., 2004), abstract semantic representations (Skipper-Kallal et al., 2015) and creativity (Mayseless et al., 2014). Hence, our results suggest that activity in the left TPJ is predominantly related to self-referential engagement, which was more effectively maintained in the context of positive-social scenes.

Interestingly, the observed modulation of functional activity and connectivity within prefrontal-temporoparietal-amygdala circuits was affected by individual characteristics that reflect emotion regulation competences and mood. We found that participants who favor emotion suppression strategies showed a weaker activation of the dmPFC and vmPFC when they engaged in positive emotions (Fig. 2C). This suggests that suppression strategies to regulate emotions may negatively impact on regulation processes that are crucial for successfully engag- ing in positive-social emotions. Suppression strategies have indeed been shown to be less efficient (the white bear effect) and less beneficial than cognitive strategies based on reappraisal (Hu et al., 2014). In fact, emotion suppression might even be counterproductive and increase stressful experiences (Gross, 2002).

An interesting result is the functional specialization of the ventral and medial prefrontal areas, TPJ and the SFG. The SFG was the only brain area that was recruited selectively during emotion regulation in a first-person perspective, irrespective of whether positive or neutral scenes were presented. Also, our meta-analytic functional characterization points to a key role of TPJ, dmPFC and SFG regions in cognitive control, as these areas were most strongly associated with the psychological category 'Cognition' and 'Social Cognition' (Fig. S1). This result accords well with imagined self-referential engagement of our participants during regulation and the social content of our stimuli. Furthermore, our connectivity analyses demonstrated that the SFG is centrally involved in top-down cognitive control processes as it responded strongly to inputs during emotion regulation, and modulated activity of the dmPFC, vmPFC and TPJ (Figs. 3 and 4). Accordingly, the SFG was also associated with the psychological categories 'Emotion' and 'Cognition' (Fig. S1). The SFG and dmPFC have previously been reported to mediate cognitive control during social-emotional appraisal and introspection (Goldberg et al., 2006; Ochsner and Gross, 2008). The $\mathrm{dmPFC}$ is also frequently engaged when evaluating social information (Amodio and Frith, 2006; Bzdok et al., 2013; Vrticka et al., 2012), situating oneself in social contexts (Amodio and Frith, 2006; Gusnard et al., 2001), and maintaining a goal-relevant regulation strategy (Gusnard et al., 2001). Unlike the SFG, the dmPFC and the vmPFC were not differentially recruited during regulation but responded mainly to the presentation of positive images (Figs. 3 and 4, Table 1). However, whereas the dmPFC and TPJ were primarily associated with the with 'Self-reflection' and 'Theory of Mind' paradigms, the vmPFC was associated with 'Competition/Cooperation', 'Reward' and 'Face Monitor/Discrimination' paradigms (Fig. S1). It thus seems likely that the vmPFC is cru- 
cially involved in stimulus valuation, or integrating the affective meaning of external stimuli with cognitive goals and/or internal representations. In line with this, previous neuroimaging and electrophysiology studies suggest that activity in the vmPFC is closely linked to behavioral measures of subjective value (Baumgartner et al., 2011; Kim and Hamann, 2007; Roy et al., 2012), and to the processing of social stimuli (Vrticka et al., 2012). This also accords well with the unique pattern of connectivity of the vmPFC to brain structures related to memory, reward, goal formation, and theory of mind (notably, the dmPFC), and to the subcortical autonomic structures like the hypothalamus and amygdala, which further suggests a crucial role of the vmPFC in affective valuation (Gusnard et al., 2001).

\subsection{Limitations}

Here we focused on engaging oneself in positive-social emotions. While this is a realistic scenario of therapeutic relevance, the current design does not allow for isolating these key aspects of emotion regulation (i.e., self-referential, social situations). This has been done elsewhere (e.g., (Vrticka et al., 2012)). Also, our study does not cover all aspects of engaging in positive emotion regulation. For example, Admon and Pizzigalli induced positive mood using humoristic cartoons which activated regions not involved in our network analyses such as the ventral striatum (Admon and Pizzagalli, 2015). Future studies might shed light on variants of positive emotion regulation and can potentially include more ROIs for more extensive network analyses. Novel DCM methods such as Bayesian model reduction (BMR) allow for computationally efficient estimation of even large network models that are in good agreement with the more conventional DCM approach applied in the present study (Friston et al., 2011, 2016; Litvak et al., 2015; Rosa et al., 2012; Seghier and Friston, 2013). Another interesting extension of our study would be the direct comparison between positive and negative emotion regulation. Our study revealed that positive emotion regulation recruited a distributed cortico-limbic network that overlaps with that involved in regulating negative emotions (Kim and Hamann, 2007; Ochsner et al., 2012; Vrticka et al., 2012), but does not allow for directly contrasting these two conditions. These future extensions will also provide important information regarding the reproducibility of the present findings and further contribute to consolidating a coherent understanding of emotion regulation. Future studies might also include more diverse participant populations to assess if the present results generalize beyond the population included in this study (which consisted predominantly of young students and researchers).

Finally, we used a multimodal approach that consisted of complementary meta-analytic (e.g., functional profiling and MACM connectivity analysis) as well as study-population-specific analyses (e.g., brain activations, DCM, questionnaires). The integration of these diverse measures is clearly complex and leaves scope for interpretation. Nevertheless, the different results do inform each other in that, for example, the meta-analytic functional profiling confirms that the study-specific brain activations are task relevant and the meta-analytic functional connectivity analysis informs which network should undergo study-specific effective connectivity analysis using DCM(Koush et al., 2019). Also, we tried combining the results we obtained to provide an integrated framework based on quantitative measures.

\subsection{Conclusion}

To better understand the neural underpinnings of positive emotion regulation in lifelike scenarios that include a self-referential perspective and social situations, we provided insights from complementary analyses including brain activations, meta-analytic functional characterization, functional and effective brain connectivity, and personality questionnaires. While there is considerable overlap between the brain re- gions involved in our task and related other designs, we aimed at integrating our converging results to describe the function and the dynamic interactions between these regions. We hope that our model of engaging oneself in positive-social emotions in healthy participants can help to identify changes in brain dynamics that prevent positive emotions in patients suffering from anxiety and mood disorders. Neurofeedback training (Sitaram et al., 2017) may then even be applied to normalize abnormal brain networks in these patients (Koush et al., 2013, 2015, 2017a, 2017b).

\section{Acknowledgements}

This study was supported by the Center for Neuroscience of the University of Geneva, the Ernest Boninchi Foundation, the Swiss National Science Foundation (YK: P300PB_161083; FS: PZ00P3-131932, PP00P2-146318, BSSG10_155915，100014_178841，32003B_166566; PV: 166704), and the Wyss Center at the Campus Biotech Geneva. FS is also supported by the Foundation for Research in Science and the Humanities at the University of Zurich (STWF-17-012), the Baugarten Stiftung, and the European Union. We thank Hanneke den Ouden for helpful discussions. The computations were performed at the EPFL on the Castor HPC cluster, and at the University of Geneva on the Baobab HPC cluster. The authors declare no competing financial interests.

\section{Appendix A. Supplementary data}

Supplementary data to this article can be found online

\section{References}

Adolphs, R., 2003. Cognitive neuroscience of human social behaviour. Nat. Rev. Neurosci. 4, 165-178.

Adolphs, R., 2009. The social brain: neural basis of social knowledge. Annu. Rev. Psychol. 60, 693-716.

Amodio, D.M., Frith, C.D., 2006. Meeting of minds: the medial frontal cortex and social cognition. Nat. Rev. Neurosci. 7, 268-277.

Ashburner, J., 2007. A fast diffeomorphic image registration algorithm. Neuroimage 38 , 95-113.

Banks, S.J., Eddy, K.T., Angstadt, M., Nathan, P.J., Phan, K.L., 2007. Amygdala - fronta connectivity during emotion regulation. Soc. Cognit. Affect Neurosci. 2, 303-312.

Baumgartner, T., Knoch, D., Hotz, P., Eisenegger, C., Fehr, E., 2011. Dorsolateral and ventromedial prefrontal cortex orchestrate normative choice. Nat. Neurosci. 14, 1468-1474.

Beck, A.T., Ward, C.H., Mendelson, M., Mock, J., Erbaugh, J., 1961. An inventory for measuring depression. Arch. Gen. Psychiatr. 4, 561-571.

Braunstein, L.M., Gross, J.J., Ochsner, K.N., 2017. Explicit and implicit emotion regulation: a multi-level framework. Soc. Cognit. Affect Neurosci. 12, 1545-1557.

Buckner, R.L., Carroll, D.C., 2007. Self-projection and the brain. Trends Cognit. Sci. 11, 49-57.

Bzdok, D., Langner, R., Schilbach, L., Engemann, D.A., Laird, A.R., Fox, P.T., Eickhoff, S.B., 2013. Segregation of the human medial prefrontal cortex in social cognition. Front. Hum. Neurosci. 7, 232.

Carpenter, S.M., Peters, E., Vastfjall, D., Isen, A.M., 2013. Positive feelings facilitate working memory and complex decision making among older adults. Cognit. Emot. 27, 184-192.

Dan-Glauser, E.S., Scherer, K.R., 2011. The Geneva affective picture database (GAPED): a new 730-picture database focusing on valence and normative significance. Behav. Res. Methods 43, 468-477.

Disner, S.G., Beevers, C.G., Haigh, E.A., Beck, A.T., 2011. Neural mechanisms of the cognitive model of depression. Nat. Rev. Neurosci. 12, 467-477.

Dunbar, R.I.M., 1998. The social brain hypothesis. Evol. Anthropol. 6, 178-190.

Eickhoff, S.B., Stephan, K.E., Mohlberg, H., Grefkes, C., Fink, G.R., Amunts, K., Zilles, K., 2005. A new SPM toolbox for combining probabilistic cytoarchitectonic maps and functional imaging data. Neuroimage 25, 1325-1335.

Etkin, A., Egner, T., Kalisch, R., 2011. Emotional processing in anterior cingulate and medial prefrontal cortex. Trends Cognit. Sci. 15, 85-93.

Fox, P.T., Laird, A.R., Fox, S.P., Fox, P.M., Uecker, A.M., Crank, M., Koenig, S.F., Lancaster, J.L., 2005. BrainMap taxonomy of experimental design: description and evaluation. Hum. Brain Mapp. 25, 185-198.

Fox, P.T., Lancaster, J.L., Laird, A.R., Eickhoff, S.B., 2014. Meta-analysis in human neuroimaging: computational modeling of large-scale databases. Annu. Rev. Neurosci. 37, 409-434.

Friston, K.J., Harrison, L., Penny, W., 2003. Dynamic causal modelling. Neuroimage 19, 1273-1302. 
Friston, K.J., Li, B., Daunizeau, J., Stephan, K.E., 2011. Network discovery with DCM. Neuroimage $56,1202-1221$.

Friston, K.J., Litvak, V., Oswal, A., Razi, A., Stephan, K.E., van Wijk, B.C.M., Ziegler, G., Zeidman, P., 2016. Bayesian model reduction and empirical Bayes for group (DCM) studies. Neuroimage 128, 413-431.

Friston, K.J., Rotshtein, P., Geng, J.J., Sterzer, P., Henson, R.N., 2006. A critique of functional localisers. Neuroimage 30, 1077-1087.

Goldberg, I.I., Harel, M., Malach, R., 2006. When the brain loses its self: prefrontal inactivation during sensorimotor processing. Neuron 50, 329-339.

Gross, J.J., 2002. Emotion regulation: affective, cognitive, and social consequences. Psychophysiology 39, 281-291.

Gross, J.J., John, O.P., 2003. Individual differences in two emotion regulation processes: implications for affect, relationships, and well-being. J. Pers. Soc. Psychol. 85, 348-362.

Gusnard, D.A., Akbudak, E., Shulman, G.L., Raichle, M.E., 2001. Medial prefrontal cortex and self-referential mental activity: relation to a default mode of brain function. Proc. Natl. Acad. Sci. U. S. A. 98, 4259-4264.

Hariri, A.R., Tessitore, A., Mattay, V.S., Fera, F., Weinberger, D.R., 2002. The amygdala response to emotional stimuli: a comparison of faces and scenes. Neuroimage 17, 317-323.

Harvey, P.O., Zaki, J., Lee, J., Ochsner, K., Green, M.F., 2013. Neural substrates of empathic accuracy in people with schizophrenia. Schizophr. Bull. 39, 617-628.

Holmes, E.A., Coughtrey, A.E., Connor, A., 2008. Looking at or through rose-tinted glasses? Imagery perspective and positive mood. Emotion 8, 875-879.

Hu, T., Zhang, D., Wang, J., Mistry, R., Ran, G., Wang, X., 2014. Relation between emotion regulation and mental health: a meta-analysis review. Psychol. Rep. 114, 341-362.

Ionta, S., Martuzzi, R., Salomon, R., Blanke, O., 2014. The brain network reflecting bodily self-consciousness: a functional connectivity study. Soc. Cognit. Affect Neurosci. 9 , 1904-1913.

Jenkinson, M., 2003. Fast, automated, N-dimensional phase-unwrapping algorithm. Magn. Reson. Med. 49, 193-197.

Kennedy, D.P., Adolphs, R., 2012. The social brain in psychiatric and neurological disorders. Trends Cognit. Sci. 16, 559-572.

Kim, S.H., Hamann, S., 2007. Neural correlates of positive and negative emotion regulation. J. Cognit. Neurosci. 19, 776-798.

Koush, Y., Ashburner, J., Prilepin, E., Sladky, R., Zeidman, P., Bibikov, S., Scharnowski, F., Nikonorov, A., De Ville, D.V., 2017a. OpenNFT: an open-source Python/Matlab framework for real-time fMRI neurofeedback training based on activity, connectivity and multivariate pattern analysis. Neuroimage 156, 489-503.

Koush, Y., Ashburner, J., Prilepin, E., Sladky, R., Zeidman, P., Bibikov, S., Scharnowski, F., Nikonorov, A., Van De Ville, D., 2017b. Real-time fMRI data for testing OpenNFT functionality. Data Brief 14, 344-347.

Koush, Y., Meskaldji, D.E., Pichon, S., Rey, G., Rieger, S.W., Linden, D.E., Van De Ville, D., Vuilleumier, P., Scharnowski, F., 2015. Learning control over emotion networks through connectivity-based neurofeedback. Cerebr. Cortex

Koush, Y., Rosa, M.J., Robineau, F., Heinen, K., S, W.R., Weiskopf, N., Vuilleumier, P., Van De Ville, D., Scharnowski, F., 2013. Connectivity-based neurofeedback: dynamic causal modeling for real-time fMRI. Neuroimage 81, 422-430.

Koush, Y., Zvyagintsev, M., Dyck, M., Mathiak, K.A., Mathiak, K., 2012. Signal quality and Bayesian signal processing in neurofeedback based on real-time fMRI. Neuroimage 59, 478-489.

Kucyi, A., Hodaie, M., Davis, K.D., 2012. Lateralization in intrinsic functional connectivity of the temporoparietal junction with salience- and attention-related brain networks. J. Neurophysiol. 108, 3382-3392.

Laird, A.R., Fox, P.M., Price, C.J., Glahn, D.C., Uecker, A.M., Lancaster, J.L., Turkeltaub, P.E., Kochunov, P., Fox, P.T., 2005. ALE meta-analysis: controlling the false discovery rate and performing statistical contrasts. Hum. Brain Mapp. 25, 155-164.

Lancaster, J.L., Woldorff, M.G., Parsons, L.M., Liotti, M., Freitas, E.S., Rainey, L., Kochunov, P.V., Nickerson, D., Mikiten, S.A., Fox, P.T., 2000. Automated Talairach Atlas labels for functional brain mapping. Hum. Brain Mapp. 10, 120-131.

Lang, P.J., Greenwald, M.K., Bradley, M.M., Hamm, A.O., 1993. Looking at pictures - affective, facial, visceral, and behavioral reactions. Psychophysiology 30, 261-273.

Lemogne, C., le Bastard, G., Mayberg, H., Volle, E., Bergouignan, L., Lehericy, S., Allilaire, J.F., Fossati, P., 2009. In search of the depressive self: extended medial prefrontal network during self-referential processing in major depression. Soc. Cognit. Affect Neurosci. 4, 305-312.

Litvak, V., Garrido, M., Zeidman, P., Friston, K., 2015. Empirical Bayes for group (DCM) studies: a reproducibility study. Front. Hum. Neurosci. 9, 670.

Marchewka, A., Zurawski, L., Jednorog, K., Grabowska, A., 2013. The Nencki Affective Picture System (NAPS): introduction to a novel, standardized, wide-range, high-quality, realistic picture database. Behav. Res. Methods

Mayseless, N., Aharon-Peretz, J., Shamay-Tsoory, S., 2014. Unleashing creativity: the role of left temporoparietal regions in evaluating and inhibiting the generation of creative ideas. Neuropsychologia 64, 157-168.

Murdaugh, D.L., Nadendla, K.D., Kana, R.K., 2014. Differential role of temporoparietal junction and medial prefrontal cortex in causal inference in autism: an independent component analysis. Neurosci. Lett. 568, 50-55.
Nadler, R.T., Rabi, R., Minda, J.P., 2010. Better mood and better performance. Learning rule-described categories is enhanced by positive mood. Psychol. Sci. 21, 1770-1776.

Oberman, L.M., Ramachandran, V.S., 2007. The simulating social mind: the role of the mirror neuron system and simulation in the social and communicative deficits of autism spectrum disorders. Psychol. Bull. 133, 310-327.

Ochsner, K.N., Gross, J.J., 2005. The cognitive control of emotion. Trends Cognit. Sci. 9, 242-249.

Ochsner, K.N., Gross, J.J., 2008. Cognitive emotion regulation: insights from social cognitive and affective neuroscience. Curr. Dir. Psychol. Sci. 17, 153-158.

Ochsner, K.N., Ray, R.R., Hughes, B., McRae, K., Cooper, J.C., Weber, J., Gabrieli, J.D., Gross, J.J., 2009. Bottom-up and top-down processes in emotion generation: common and distinct neural mechanisms. Psychol. Sci. 20, 1322-1331.

Ochsner, K.N., Silvers, J.A., Buhle, J.T., 2012. Functional imaging studies of emotion regulation: a synthetic review and evolving model of the cognitive control of emotion. Year Cognit. Neurosci. 1251, E1-E24.

Penny, W.D., Stephan, K.E., Daunizeau, J., Rosa, M.J., Friston, K.J., Schofield, T.M., Leff, A.P., 2010. Comparing families of dynamic causal models. PLoS Comput. Biol. 6.

Reeck, C., Ames, D.R., Ochsner, K.N., 2016. The social regulation of emotion: an integrative, cross-disciplinary model. Trends Cognit. Sci. 20, 47-63.

Rosa, M.J., Friston, K., Penny, W., 2012. Post-hoc selection of dynamic causal models. J. Neurosci. Methods 208, 66-78.

Roy, M., Shohamy, D., Wager, T.D., 2012. Ventromedial prefrontal-subcortical systems and the generation of affective meaning. Trends Cognit. Sci. 16, 147-156.

Samson, D., Apperly, I.A., Chiavarino, C., Humphreys, G.W., 2004. Left temporoparietal junction is necessary for representing someone else's belief. Nat. Neurosci. 7, 499-500.

Saxe, R., Kanwisher, N., 2003. People thinking about thinking people. The role of the temporo-parietal junction in "theory of mind". Neuroimage 19, 1835-1842.

Scherer, K.R., 1982. Emotion as a process: function, origin and regulation. Soc. Sci. Inf. 21, 555-570.

Schlosser, R.G., Wagner, G., Koch, K., Dahnke, R., Reichenbach, J.R., Sauer, H., 2008 Fronto-cingulate effective connectivity in major depression: a study with fMRI and dynamic causal modeling. Neuroimage 43, 645-655.

Seghier, M.L., Friston, K.J., 2013. Network discovery with large DCMs. Neuroimage 68, $181-191$.

Sitaram, R., Ros, T., Stoeckel, L., Haller, S., Scharnowski, F., Lewis-Peacock, J., Weiskopf, N., Blefari, M.L., Rana, M., Oblak, E., Birbaumer, N., Sulzer, J., 2017. Closed-loop brain training: the science of neurofeedback. Nat. Neurosci. Rev.

Skipper-Kallal, L.M., Mirman, D., Olson, I.R., 2015. Converging evidence from fMRI and aphasia that the left temporoparietal cortex has an essential role in representing abstract semantic knowledge. Cortex 69, 104-120.

Spreng, R.N., Mar, R.A., Kim, A.S., 2009. The common neural basis of autobiographical memory, prospection, navigation, theory of mind, and the default mode: a quantitative meta-analysis. J. Cognit. Neurosci. 21, 489-510.

Stephan, K.E., Penny, W.D., Moran, R.J., den Ouden, H.E., Daunizeau, J., Friston, K.J., 2010. Ten simple rules for dynamic causal modeling. Neuroimage 49, 3099-3109.

Teufel, C., Fletcher, P.C., Davis, G., 2010. Seeing other minds: attributed mental states in fluence perception. Trends Cognit. Sci. 14, 376-382.

Treadway, M.T., Zald, D.H., 2011. Reconsidering anhedonia in depression: lessons from translational neuroscience. Neurosci. Biobehav. Rev. 35, 537-555.

Triantafyllou, C., Polimeni, J.R., Wald, L.L., 2011. Physiological noise and signal-to-noise ratio in fMRI with multi-channel array coils. Neuroimage 55, 597-606.

van der Velden, A.M., Kuyken, W., Wattar, U., Crane, C., Pallesen, K.J., Dahlgaard, J., Fjorback, L.O., Piet, J., 2015. A systematic review of mechanisms of change in mindfulness-based cognitive therapy in the treatment of recurrent major depressive disorder. Clin. Psychol. Rev. 37, 26-39.

Van Dijk, K.R., Sabuncu, M.R., Buckner, R.L., 2012. The influence of head motion on intrinsic functional connectivity MRI. Neuroimage 59, 431-438.

Vrticka, P., Sander, D., Vuilleumier, P., 2012. Lateralized interactive social content and valence processing within the human amygdala. Front. Hum. Neurosci. 6, 358.

Wagner, G., Schachtzabel, C., Peikert, G., Bar, K.J., 2015. The neural basis of the abnormal self-referential processing and its impact on cognitive control in depressed patients. Hum. Brain Mapp. 36, 2781-2794.

Watson, D., Clark, L.A., Tellegen, A., 1988. Development and validation of brief measures of positive and negative affect: the PANAS scales. J. Pers. Soc. Psychol. 54, 1063-1070.

Weiskopf, N., Hutton, C., Josephs, O., Deichmann, R., 2006. Optimal EPI parameters for reduction of susceptibility-induced BOLD sensitivity losses: a whole-brain analysis at $3 \mathrm{~T}$ and $1.5 \mathrm{~T}$. Neuroimage 33, 493-504.

Weiskopf, N., Hutton, C., Josephs, O., Turner, R., Deichmann, R., 2007. Optimized EPI for fMRI studies of the orbitofrontal cortex: compensation of susceptibility-induced gradients in the readout direction. Magn. Reson. Mater. Phys. Biol. Med. 20, 39-49.

Yarkoni, T., Poldrack, R.A., Nichols, T.E., Van Essen, D.C., Wager, T.D., 2011. Large-scale automated synthesis of human functional neuroimaging data. Nat. Methods 8 , 665-U695.

Zaki, J., Ochsner, K.N., 2012. The neuroscience of empathy: progress, pitfalls and promise. Nat. Neurosci. 15, 675-680. 